\title{
Dominique CARDON, Culture numérique
}

Sébastien Shulz

\section{(2) OpenEdition}

\section{Journals}

Édition électronique

URL : http://journals.openedition.org/ress/5374

DOI : $10.4000 /$ ress. 5374

ISBN : $1663-4446$

ISSN : 1663-4446

Éditeur

Librairie Droz

\section{Édition imprimée}

Date de publication : 20 décembre 2019

Pagination : 312-316

ISSN : 0048-8046

Référence électronique

Sébastien Shulz, "Dominique CARDON, Culture numérique », Revue européenne des sciences sociales [En ligne], 57-2 | 2019, mis en ligne le 20 décembre 2019, consulté le 01 décembre 2020. URL : http:// journals.openedition.org/ress/5374; DOI : https://doi.org/10.4000/ress.5374

Ce document a été généré automatiquement le 1 décembre 2020

(C) Librairie Droz 


\title{
Dominique CARDON, Culture numérique
}

\author{
Sébastien Shulz
}

\section{RÉFÉRENCE}

Dominique CARDON, 2019, Culture numérique, Paris, Les Presses de Sciences Po, 432 p.

1 Avec Culture numérique, Dominique Cardon nous livre la synthèse de vingt années d'observations, d'enseignements et de recherches menées sur les mondes numériques. Tiré d'un cours qu'il donne à Sciences Po, ce livre d'introduction est un événement éditorial important pour la diffusion des Internet studies. Trente ans après la naissance $\mathrm{du}$ Web, les premières pages stabilisées de l'histoire de «la grande aventure du numérique » (p. 420) semblent pouvoir être écrites. Cardon y parvient avec une clarté qu'il faut lui reconnaître sur un sujet aussi touffu, et qui le distingue d'autres ouvrages similaires. Les néophytes, les journalistes et les étudiants trouveront dans ce livre un tableau (presque) complet des grands thèmes de la culture numérique, avec des références aussi variées qu'utiles à la fin de chaque chapitre. Pour les chercheurs, il représente un outil de synthèse heuristique et pluridisciplinaire (histoire, économie, sciences politiques, études des sciences et des techniques) dont l'utilité repose moins sur une recension exhaustive des plus récentes enquêtes sur le numérique que sur un vrai travail de cartographie et de catégorisation de l'espace numérique, à travers notamment de nombreuses matrices. Suivant un parcours chronologique en six parties, l'ouvrage s'ouvre sur une généalogie d'Internet (partie 1) et du Web (partie 2) avant de s'intéresser, dans une perspective plus sociologique, aux réseaux sociaux (partie 3), puis aux dimensions politique du numérique (partie 4), économique des plates-formes (partie 5) et algorithmique des big data (partie 6).

2 Cardon commence son ouvrage en retraçant l'histoire socio-technique de l'informatique et d'Internet. Il relate la mise en place de la communication par paquets, des fameux protocoles TCP/IP et de l'architecture distribuée en réseau qui a abouti à 
l'infrastructure mère de l'Internet d'aujourd'hui. Il rappelle l'origine militaire du financement de ces premières recherches tout en insistant sur l'esprit de libre coopération entre pairs qui s'y développe et qui constituera l'un des modes de fonctionnement caractéristique de la culture numérique, comme en témoignent les logiciels libres et la gouvernance partagée d'Internet. Au tournant des années 1970, la contre-culture va marquer durablement l'histoire d'Internet en assignant à l'informatique personnelle et au réseau des réseaux les vertus d'autonomie, de créativité et d'émancipation individuelle et collective qui caractérisent «l'esprit hacker » face au monde monotone, industriel et militarisé de la guerre froide. Dans le bouillonnement californien de ces années, des chercheurs comme Douglas Engelbart ainsi que des bidouilleurs comme Steve Wozniak vont contribuer à forger le mythe d'un cyberespace affranchissant les humains des carcans du monde réel en développant la micro-informatique et en formant les premières communautés virtuelles. L'homogénéité sociologique de ces communautés, ou encore la promotion du mérite individuel et de l'esprit entrepreneurial contrasteront néanmoins avec cette prétention égalitariste : une ambivalence qui traverse jusqu'à nos jours la culture numérique.

3 Le second chapitre expose l'apparition en 1990 des liens hypertextes et du web, véritable révolution classificatoire qui permet aux internautes de se connecter entre eux et de trouver facilement les contenus en ligne. C'est la naissance des navigateurs et de l'Internet grand public. Les tenants de "la nouvelle économie» y voient une incroyable source de croissance économique. Les start-up comme Yahoo! deviennent rapidement survalorisées tandis que les géants industriels tentent de ne pas perdre la main en procédant à des rachats massifs, une course qui aboutira à l'éclatement de la bulle Internet en 2000. En fait, la particularité du Web tient à sa capacité à faire émerger de la valeur, de l'innovation et de l'intelligence collective par une coopération venant de la base. L'innovation ascendante que Cardon prend comme exemple vient souvent d'un problème personnel, d'une solution bidouillée, partagée puis reprise et améliorée dans un cercle vertueux (sous certaines conditions) par des individus qui forment une communauté ad hoc. Certains, comme les grandes plates-formes, captent cette valeur. D'autres, plus proches de l'éthique hacker, revendiquent qu'elle soit repartagée à la communauté qui en est à l'origine. Des militants et universitaires comme Lawrence Lessig, inventeur des licences Creative Commons, défendent ainsi les biens communs numériques comme des ressources ouvertes et inappropriables. Wikipedia, auquel Cardon consacre un sous-chapitre, est l'exemple type de commun numérique qui, en partant d'une initiative personnelle, a réussi à agréger des millions de contributions grâce à des règles élaborées par une communauté pour en faire émerger une intelligence collective dont les fruits ont été mis à la disposition à tous.

4 La troisième partie, plus sociologique, s'intéresse à la structure du web et à ce qu'y font les internautes. D'abord, le Web élargit l'espace public en le rendant plus populaire et interactif. Au-delà de l'élite professionnelle qui reste malgré tout la plus écoutée, des amateurs y prennent massivement la parole : les «quidams parlent de quidams à des quidams » (p. 150). De nouveaux régimes de visibilité se mettent ainsi en place en deçà des gatekeepers (intermédiaires) traditionnels. Ensuite, le Web 2.0 engage de nouvelles formes de sociabilité. S'il sert principalement à prolonger les interactions avec notre cercle proche, sa spécificité est de permettre d'entrer en contact avec des inconnus et de maintenir des liens faibles, notamment sur les réseaux sociaux. Cardon typologise ces derniers selon la représentation de l'identité qu'on y expose (réelle ou imaginaire), et le degré de visibilité du contenu qu'on y diffuse (fermé ou ouvert). Concernant 
l'identité numérique, l'auteur tire les leçons des récentes recherches: en ligne, l'exposition de soi est généralement motivée par un désir de reconnaissance, reflète notre profil sociologique hors-ligne et relève de stratégies loin d'être naïves pour délimiter notre vie privée. Concernant le contenu, le Web a finalement entrainé l'émergence de pratiques créatives originales allant du remix réalisé par des fans aux vidéos d'humoristes amateurs. Pour autant, même si cette expressivité s'est popularisée, elle est aujourd'hui principalement exploitée par les plus dotés en capital social et culturel et certaines entreprises. D'autres contenus plus sensibles circulent sur la toile, et le chapitre se clôt sur les enjeux soulevés par leur régulation.

5 La quatrième partie du livre analyse ce que font les outils numériques à la sphère publique et politique. D'abord, la culture politique des collectifs numériques - ou issues du numérique comme ceux du printemps arabe ou des gilets jaunes - se caractérise par l'horizontalité, le refus de porte-parole et la préférence donnée aux débats sur les procédures plutôt qu'aux programmes. C'est ce que Cardon nomme la démocratie Internet. Ensuite, d'un impact limité sur les acteurs traditionnels de la démocratie représentative qui s'en servent pour affiner leurs stratégies de communication, les outils numériques ont surtout été utilisés par des mouvements contestataires et de vigilance citoyenne. Les résultats décevants des procédures de participation en ligne mises en place par les institutions publiques contrastent avec les formes plus spontanées et virales d'expressivité numérique dont les hastags sont caractéristiques. Enfin, bien que la télévision reste la première source d'information, les médias ont dû s'ajuster à ces nouvelles dynamiques de l'espace public. Deux tendances du journalisme en ligne se sont dessinées : d'un côté un journalisme du buzz, courant derrière l'audience pour rentabiliser des placements publicitaires à faible coût ; d'un autre, un journalisme de qualité, enquêtant parfois de manière innovante sur des données publiques ouvertes (open data) ou ayant fuité (leaks), et fonctionnant via des systèmes de paiement modulable avec des contenus gratuits et d'autres réservés aux abonnés. Quant aux fakenews, Cardon rappelle utilement que si des informations douteuses peuvent circuler dans des «niches de bavardage à faible visibilité » (p. 274), les médias traditionnels peuvent se défendre - encore faut-il le vouloir - contre une désinformation qui ne touchera ainsi pas ou peu le grand public. Le chapitre se conclut sur un tour d'horizon des civic-tech, ces outils mis en place par des collectifs citoyens pour perfectionner, enrichir ou compléter la démocratie représentative tout en soulignant leur impact limité, l'idéalisme un peu naïf et l'homogénéité sociologique des acteurs qui les portent.

6 L'avant-dernière partie du livre expose les rouages économiques des plates-formes. Comment expliquer que les fameux GAFA (Google, Amazone, Facebook, Apple) engendrent des records de capitalisation boursière et des bénéfices sans précédent ? Premièrement, plusieurs lois, dont «l'effet de réseau » qui augmente la valeur d'usage en fonction du nombre d'usagers, expliquent la tendance monopolistique des industries numériques. Deuxièmement, ces dernières fonctionnent selon un modèle « biface » où la plate-forme numérique orchestre la rencontre entre une offre et une demande: généralement gratuite pour attirer les utilisateurs (qui génèrent une externalité positive via l'effet de réseau) les plates-formes font reposer les coûts sur l'autre face du marché, à des fournisseurs de service ou des régies publicitaires. Le marché publicitaire a ainsi largement soutenu l'économie numérique dans son ensemble et les géants du Web en particulier. Google aspire $50 \%$ du marché mondial de la publicité en ligne grâce aux cookies et aux données personnelles que la plate-forme récolte (malgré une réticence 
croissante de la part des usagers et des régulateurs) ainsi qu'à un ingénieux système d'enchères auquel prennent part les régies publicitaires pour afficher de manière ciblée leur contenu. Troisièmement, pour bien fonctionner, certaines plates-formes d'intermédiation comme Airbnb ont dû convaincre les utilisateurs qu'il n'y avait pas de risques à acheter un bien, un service, ou à louer l'appartement d'un inconnu. Ce sont principalement les dispositifs de notation et d'avis qui ont joué ce rôle de confiance: $96 \%$ des acheteurs en ligne y sont attentifs, bien qu'il existe des risques de manipulation. Quatrièmement, l'État contribue à cette économie des plates-formes en mettant à disposition des données publiques. Les expériences récentes montrent que seuls les grands acteurs du numériques sont capables de surmonter les difficultés techniques inhérentes à la réutilisation des données massives et hétérogènes ouvertes par les administrations. Finalement, les plates-formes ont fait émerger ce que certains analystes appellent le digital labour. Que ce soit en capitalisant sur nos propres activités en ligne, en payant des sommes dérisoires à des individus souvent marginalisés du marché de l'emploi pour qu'ils réalisent des micro-tâches alimentant une intelligence artificielle ou encore en incitant la flexibilité de l'auto-entrepreneur tout en contrôlant son activité, les plates-formes ont révélé de nouveaux gisements d'activité tout en jetant un flou sur la question du travail et de sa protection, que Cardon appelle à réguler.

7 Le dernier chapitre traite de l'abondance des données (big data) et des algorithmes créés pour les traiter. Cardon distingue quatre familles d'algorithmes qui tentent de baliser l'immense dédale du Web. La première famille classe les contenus en fonction de leur popularité, c'est-à-dire en fonction de l'affluence d'une page. La seconde famille les classe par rapport à leur autorité, déterminée en fonction du nombre d'hyperliens qui renvoient vers eux. Caractéristique du moteur de recherche de Google (pagerank), la logique classificatoire derrière cet algorithme est que plus une page est citée par un grand nombre d'internautes, plus elle est digne d'intérêt et possède une certaine autorité sur un sujet donné. Par rapport aux deux premières, les deux familles suivantes adaptent leur résultat aux caractéristiques de l'utilisateur. La troisième s'attache ainsi à mesurer la réputation d'un contenu à travers des métriques de popularité. Il est intéressant de noter qu'elle engendre des stratégies d'influence, déployée par des marques comme par nous-mêmes, pour colorier l'espace social numérique selon certaines préférences via les likes et les re-tweet. Finalement, la dernière famille ne s'appuie plus sur l'affichage de nos préférences, mais sur les traces de notre activité numérique, jugées plus fidèles à notre véritable identité: en les comparant à celles d'autres utilisateurs, les algorithmes de cette famille, caractéristique d'Amazon, prédisent nos comportements futurs. Cardon relate ensuite l'histoire du mythe et des technologies de l'intelligence artificielle, en expliquant le fonctionnement de l'apprentissage profond (deep learning) et les risques inhérents à un mode de calcul dont nous ne connaissons pas les rouages et qui pourtant est amené à avoir des impacts majeurs sur nos vies. Il en appelle à un audit des algorithmes en fonction de l'intentionnalité des concepteurs et de leurs effets indésirables. Le livre s'achève sur une réflexion autour de la surveillance numérique exercée par le marché, l'État et les autres internautes, ainsi que les marges de liberté que nous devons défendre.

8 De l'Internet émancipateur à la surveillance de masse, des mythes fondateurs jusqu'à l'actualité brûlante, le livre traverse les grands thèmes de la culture numérique avec une exigence de synthèse qui rend la lecture aussi excitante que marquante et la 
distingue d'autres livres d'introduction aux Internet studies plus plats ou scolaires. On pourra regretter le traitement parfois mal ajusté de certains sujets dans l'économie générale du livre, notamment l'open data dans la section sur l'économie des platesformes, ainsi que certains enjeux trop rapidement éludés. Mais comme nous y invite Cardon, il incombe au lecteur de poursuivre par lui-même l'exploration de ces enjeux dans d'autres ouvrages plus spécialisés... et sur Internet !

\section{AUTEURS}

\section{SÉBASTIEN SHULZ}

Université Paris-Est Marne-la-Vallée, Lisis 